\title{
Analyst
}

Cite this: Analyst, 2014, 139, 1372

\section{Oxidized polyethylene films for orienting polar molecules for linear dichroism spectroscopy $\dagger$}

\author{
Kasra Razmkhah, Nikola Paul Chmel, ${ }^{*}$ Matthew I. Gibson and Alison Rodger
}

Stretched polyethylene (PE) films have been used to orient small molecules for decades by depositing solutions on their surface and allowing the solvent to evaporate leaving the analyte absorbed on the polymer film. However, the non-polar hydrophobic nature of PE is an obstacle to aligning polar molecules and biological samples. In this work PE film was treated with oxygen plasma in order to increase surface hydrophilicity. Different treatment conditions were evaluated using contact angle measurement and $X$-ray photoelectron spectroscopy. Treated $P E\left(P E^{O X}\right)$ films are shown to be able to align molecules of different polarities including progesterone, 1-pyrenecarboxaldehyde, 4',6-diamidino2-phenylindole (DAPI) and anthracene. The degree of alignment of each molecule was studied by running series of linear dichroism (LD) experiments and the polarizations of electronic transition moments were determined. For the first time optimal conditions (such as stretching factor and concentration of the sample) for stretched film LD were determined. PE ${ }^{\mathrm{OX}}$ aligning ability was compared to that of normal PE films. Progesterone showed a slightly better alignment on $\mathrm{PE}^{\mathrm{OX}}$ than $\mathrm{PE}$. 1-Pyrenecarboxaldehyde oriented differently on the two different films which enabled transition moment assignment for this low symmetry molecule. DAPI (which does not align on PE) aligned well on $\mathrm{PE}^{\mathrm{OX}}$ and enabled us to obtain better LD data than had previously been collected with polyvinyl alcohol. Anthracene alignment and formation of dimers and higher order structures were studied in much more detail than previously possible, showing a variety of assemblies on $\mathrm{PE}$ and $\mathrm{PE} \mathrm{OX}^{\mathrm{X}}$ films.

Received 16th December 2013 Accepted 20th January 2014

DOI: $10.1039 / c 3 a n 02322 b$

www.rsc.org/analyst

$$
\mathrm{LD}=A_{Z}-A_{Y}
$$

\section{Introduction}

Planar aromatic molecules are seeing increasing applications as spectroscopic labels in optical microscopy and spectroscopy, particularly to probe the behaviour of complex biomolecular assemblies. If one merely wishes to see where a label is and perhaps how its concentration changes in time by analysing images for localisation or dynamics of cellular components or in FRAP (fluorescence recovery after bleaching) experiments, a coloured label, usually with a good quantum yield, is all that is required. However, if one is trying to extract geometric information from an experiment, as we do in flow linear dichroism experiments, more information about transition polarizations and changes in probe spectroscopy with probe-probe interactions are required. The aim of this work was to develop methods to help us understand the spectroscopy of potential probe molecules.

Linear dichroism (LD) is the difference in absorbance, $A$, of light polarized parallel $(Z)$ and perpendicular $(Y)$ to the orientation direction

Department of Chemistry and Warwick Analytical Science Centre, University of Warwick, Coventry, CV4 7AL, UK. E-mail: N.Chmel@warwick.ac.uk; Fax: +44 2476575795; Tel: +44 2476574696

$\dagger$ Electronic supplementary information (ESI) available: Additional experimental details and data, and derivation of the equations used. See DOI: 10.1039/c3an02322b
Flow LD can be used to determine relative orientations of molecules, such as DNA-binding ligands and DNA bases, ${ }^{\mathbf{1 - 6}}$ or peptides and lipids in liposomes, ${ }^{7-9}$ or nucleotides and proteins in fibrous proteins such as FtsZ $^{10}$ or tubulin, ${ }^{11}$ or molecules binding to carbon nanotubes. ${ }^{12}$ More recently we have been attaching fluorescent probes to proteins on viruses and proteins that make up the bacterial cell division machinery and have been surprised how little spectroscopic information is available in the literature on the transition polarizations of molecules routinely used as optical probes. In a recent paper we determined the polarizations of the accessible UV absorbance bands of fluorene and the Fmoc group using stretched polyethylene (PE) films and used the results to interpret flow LD data collected on peptide fibres including the Fmoc chromophore. ${ }^{13}$

We have previously been precluded from following a similar approach for many of the molecules in which we are interested because they are too hydrophilic to be oriented on stretched PE films made from commercial sandwich-bag PE films (our preferred substrate).$^{14}$ Although we have previously collected stretched film data on polyvinylalcohol (PVA) films, these films take days to prepare and usually involve the analyte being integrated into the polymerising film. ${ }^{15}$ This makes the kind of experiments discussed below, where we vary concentration of 
parent solution and degree of stretch, extremely time consuming. PVA films are also very brittle and hard to stretch reproducibly. ${ }^{\mathbf{1 4}}$

There has been discussion in the literature about the mechanisms that operate to cause small molecules to orient on PE films. Thulstrup et al. in $1970^{16}$ concluded that it was generally agreed that most of the solute was dissolved in the amorphous parts of a PE sheet. Despite this, Wirtz et al. ${ }^{17}$ concluded in 2011 that the oriented chromophores are bound to PE crystals (though with a distribution about the stretch direction) and are oriented when the crystallites to which they are bound orient. They also deduced that the crystallites become oriented along the stretching direction of a film, because the large local strain destroys the lamellae (thin extended sheets of crystallites that are typically tens of nanometers thick and tens of micrometers long) and subsequently recrystallization occurs. Thus they concluded that stretching does not cause a gradual increase in alignment of a molecule but causes the crystallite to which it is bound to orient or, at higher stretch ratios, a phase change that leads to more crystallites. We do know that molecules are likely to orient on PE with their long axes parallel to the stretching direction. ${ }^{18}$

In this paper we report the development and application of oxidised polyethylene films $\left(\mathrm{PE}^{\mathrm{OX}}\right)$ to determine transition polarizations of polar molecules with the same versatility and ease of use that is possible with PE. We illustrate its utility by considering the spectroscopy of progesterone, 1-pyrenecarboxaldehyde (PyCO), 4',6-diamidino-2-phenylindole (DAPI) and anthracene. Contrary to our expectations, the $D_{2 \mathrm{~h}}$ symmetric hydrocarbon anthracene proved to be the most challenging due to its propensity to assemble into higher order structures. Our results show that $\mathrm{PE}^{\mathrm{OX}}$ behaves in much the same as PE except that it attracts hydrophilic molecules. We have also made some further progress in understanding the nature of the films.

\section{Materials and methods}

\section{Materials}

All chemicals were purchased from Sigma-Aldrich and used without further purification. Solvents used in the measurements were 18.2 M $\Omega$ water (Millipore Direct-QTM), chloroform (spectrophotometric grade, Sigma-Aldrich), methanol (Laboratory grade, Fisher Chemicals) and methyl cyclohexane (spectrophotometric grade, Sigma-Aldrich). The PE films to be used as sample matrices were cut from Glad ${ }^{\circledR}$ Snap Lock $®$ bags, and stretched so that the direction of stretch is parallel to the observable faint lines in the film. Stock solutions of each analyte were prepared in chloroform (for $\mathrm{PE}$ ) or methanol (for $\mathrm{PE}^{\mathrm{OX}}$ ).

\section{PE ${ }^{\mathrm{OX}}$ oxidised films}

PE sheets with dimensions of $2.5 \times 4 \mathrm{~cm}$ were placed in an Emitech K1050X Plasma Asher connected to an oxygen gas supply for $1 \mathrm{~min}$ at $50 \mathrm{~W}$ power setting.

\section{Sample preparation}

For most experiments, a piece of $\mathrm{PE}$ or $\mathrm{PE}^{\mathrm{OX}}$ film (of size $2.5 \times$ $4 \mathrm{~cm}^{2}$ ) was placed between two jaws of the mechanical stretcher designed for this purpose (Fig. 1a). The film was then stretched from 2.5 centimetres to different lengths. The analyte was sometimes added before and sometimes after stretching the film (see details in Results and discussion). Baselines were collected on stretched films where a small volume of solvent had been dropped on and left to dry. Samples $(30-40 \mu \mathrm{L}$ in solution with the same solvent as the baseline) were placed on the stretched film, then left to evaporate creating a dried layer. The stretched film and its holder were usually placed horizontally into the spectrometer so that the light beam was incident on the analyte with the stretch direction was horizontal (in our instrument the direction of the parallel polarized light).

In order to check for the presence of higher order structures e.g. formation of dimers or sample crystallisation on the surface of the film, several samples were routinely prepared using serial dilutions of the stock solution. In the absence of higher order structures, shapes of the obtained LD spectra are independent of concentration.

In some experiments the film was mounted in a cylindrical holder (Fig. 1b) and rotated to different orientations. For these

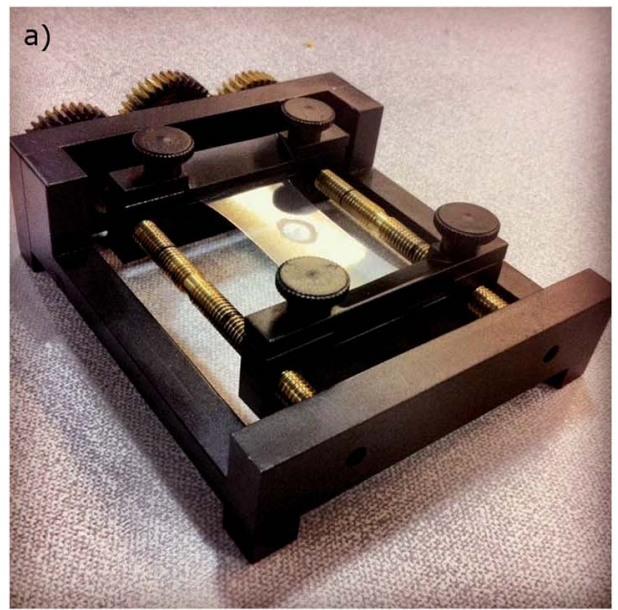

b)

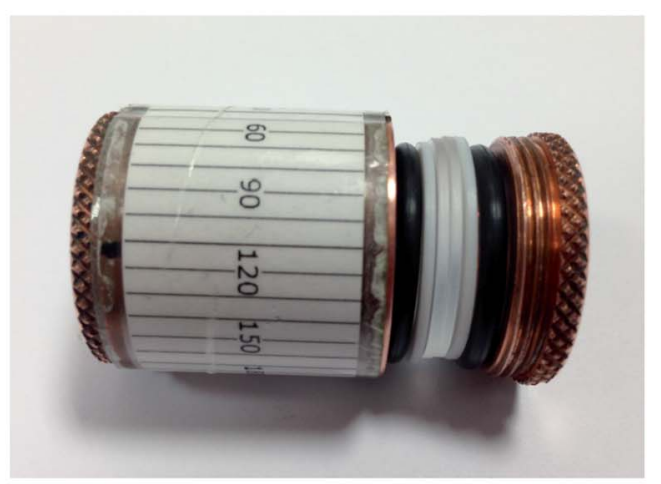

Fig. 1 (a) Mechanical film stretcher designed and built at the University of Warwick. (b) Cylindrical film holder (originally designed for oriented circular dichroism measurements) ${ }^{19}$ where film is held between two quartz plates ( $1 \mathrm{~mm}$ thick) themselves sandwiched between Teflon rings and $\mathrm{O}$-rings. 
film-rotation LD experiments, the film was first stretched in the film stretcher, the sample added and then the film transferred (ensuring it remained stretched) to the cylindrical film holder shown in Fig. 1b. To secure the sample within the cylindrical film holder, the two quartz plates were placed on either side of the PE film and clamped to maintain the tension on film. The film was cut to remove excess PE film. The sandwiched film was then placed in the holder and fixed in place by a screw fitting which, when screwed fully into the holder, maintained the tension on the film.

\section{Ultra-violet absorbance and linear dichroism spectroscopy}

Ultra-violet absorbance and LD spectra on films oriented with the stretch direction parallel to the horizontally polarized light were measured using a JASCO J-815 circular dichroism spectropolarimeter adapted for LD spectroscopy by collecting LD in one data channel and $\mathrm{A}$ in another. In general, baselines were collected on the films before the addition of the sample. The data acquisition and baseline subtraction were performed using the J-815 Spectra Manager software version 2.07.02. The spectra of samples (and baselines) were measured as a function of different stretching lengths of polyethylene. If a single film was measured at different stretch factors, baselines were measured on three independent films treated in as close to identical fashion as was possible. All measurements for film-rotation LD were performed using a Bio-Logic MOS-450 spectrometer.

Reduced $\mathrm{LD}\left(\mathrm{LD}^{\mathrm{r}}\right)$, the $\mathrm{LD}$ divided by the isotropic absorbance, is independent of concentration and path length (sample thickness, which can change upon stretching). The usefulness of the $\mathrm{LD}^{\mathrm{r}}$ depends on both spectra being measured on exactly the same molecules. This requires both the film not to be moved and there not to be any unoriented molecules in the light beam whose absorbance is measured but which do not contribute to the LD. In the simple case of uniaxial orientation with the horizontal polarization of the instrument along the film stretch direction ${ }^{\mathbf{1 4}}$

$$
\mathrm{LD}^{\mathrm{r}}=\frac{\mathrm{LD}}{A}=\frac{3}{2} S\left(3\left\langle\cos ^{2} \alpha\right\rangle-1\right)
$$

where $\alpha$ is the angle between the molecular orientation direction, $z$, and the polarization of the transition of interest, \langle\rangle denotes average over the population of molecules present, and $S$ is the orientation parameter ( 0 for unoriented samples, 1 for perfectly oriented samples).

\section{X-ray photoemission spectroscopy}

The X-ray photoemission spectroscopy (XPS) data ${ }^{20}$ were collected at the Science City Photoemission Facility, University of Warwick. The samples investigated in this study were mounted on Omicron sample plates using electrically conductive carbon tape and loaded in to the fast-entry chamber. Once a pressure of less than $1 \times 10^{-7} \mathrm{mbar}$ had been achieved (approx. 1 hour), the samples were transferred to a 12-stage storage carousel, located between the preparation and main analysis chambers, for storage at pressures of less than $2 \times 10^{-10}$ mbar. XPS measurements were conducted in the main analysis chamber (base pressure $2 \times 10^{-11} \mathrm{mbar}$ ), with the sample being illuminated using an XM1000 monochromatic Al-K $\alpha$ X-ray source (Omicron Nanotechnology). The measurements were conducted at room temperature and at a take-off angle of $90^{\circ}$ (more penetration depth) and $30^{\circ}$ (closer to the surface). The photoelectrons were detected using a Sphera electron analyser (Omicron Nanotechnology), with the core levels recorded using a pass energy of $10 \mathrm{eV}$ (resolution approx. $0.47 \mathrm{eV}$ ). Due to the insulating nature of the samples, a CN10 charge neutralizer (Omicron Nanotechnology) had to be used in order to prevent surface charging, whereby a low energy (typically $1.5 \mathrm{eV}$ ) beam of electrons was directed on to the sample during XPS data acquisition. The data were analysed using the CasaXPS package, using Shirley backgrounds and mixed Gaussian-Lorentzian (Voigt) line shapes. All binding energies were calibrated to the $\mathrm{C} 1 \mathrm{~s}$ peak at $284.6 \mathrm{eV}$. The energy is calibrated weekly using the Fermi edge of a polycrystalline $\mathrm{Ag}$ sample is accurate to $( \pm) 0.02 \mathrm{eV}$. Compositional analysis of $\mathrm{Au}$, $\mathrm{Cu}$ and $\mathrm{Ag}$ samples was used to give a transmission function for the analyser resulting in an accuracy of around $1 \%$.

\section{Contact angle measurements}

Contact angle measurements were performed using a Krüss DSA (droplet shape analyser) 100 (Krüss Instruments, Germany) on a $10 \mu \mathrm{L}$ droplet of water dispensed onto polyethylene substrate. The Tangent-1 fitting measurement was used to yield the left and right three phase contact angles of the droplet.

\section{XRD measurements}

X-ray diffraction (XRD) experiments were performed on Siemens D500 diffractometer with the $\operatorname{CuK} \alpha(\lambda=1.5428 \AA)$ radiation source, step $0.02^{\circ}$ and step interval $20 \mathrm{~s}$.

\section{Results and discussion}

Linear dichroism, LD, is a differential absorption spectroscopy technique from which one can extract transition moment polarizations if the sample orientation is known or chromophore orientation information if transition moments have been assigned. The main work of this paper relates to a new polymer surface for $\mathrm{LD}, \mathrm{PE}^{\mathrm{OX}}$. The results below describe how $\mathrm{PE}^{\mathrm{OX}}$ was produced and characterised thus enabling us to collect data on previously intractable samples.

\section{Producing and characterising non-polar and polar PE films}

Tissington, et al., ${ }^{21}$ showed that by using oxygen plasma treatment it is possible to form active oxygen containing groups on the surface of the polyethylene film. In this work pieces of PE film were placed in a Plasma Asher and treated with two different powers (50 and $100 \mathrm{~W}$ ) and four different timings (10, 30, 60 and 120 seconds). As Fig. 2 shows, the hydrophilicity of the surface increases with the duration of the treatment. However, the lower power showed higher final levels of hydrophilicity as deduced from the water contact angle (Fig. 2). This could be caused by the degradation of the PE fibres at high plasma power. ${ }^{22}$ For our spectroscopy experiments we therefore prepared films using $50 \mathrm{~W}$ and 1 minute and denote the treated films as $\mathrm{PE}^{\mathrm{OX}}$. 
a)

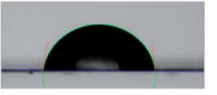

$50 \mathrm{~W}-10 \mathrm{~s}$

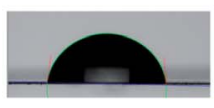

$100 \mathrm{~W}-10 \mathrm{~s}$

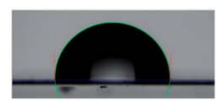

Untreated PE film

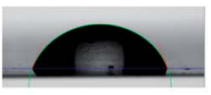

$50 \mathrm{~W}-30 \mathrm{~s}$

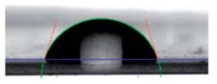

$100 \mathrm{~W}-30 \mathrm{~s}$

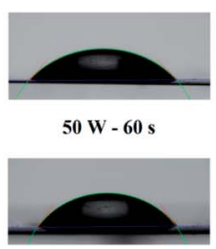

$100 \mathrm{~W}-60 s$

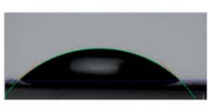

$50 \mathrm{~W}-120 \mathrm{~s}$

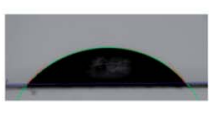

$100 \mathrm{w}-120 \mathrm{~s}$ b)

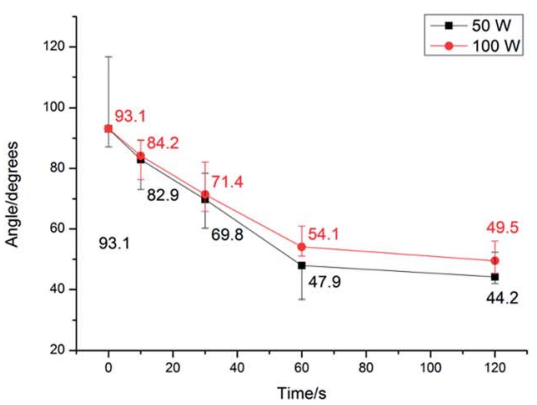

Fig. 2 (a) Water drops on polyethylene films treated at the indicated power levels for the indicated times. (b) The contact angle of PE films placed in the plasma asher at different power levels as a function of time.

Table 1 Carbon $1 \mathrm{~s}$ orbital XPS data for $\mathrm{PE}$ and $\mathrm{PE}^{\mathrm{OX}}$. $30^{\circ}$ denotes $30^{\circ}$ angle of beam incidence. $90^{\circ}$ denotes perpendicular incidence (so maximum penetration)

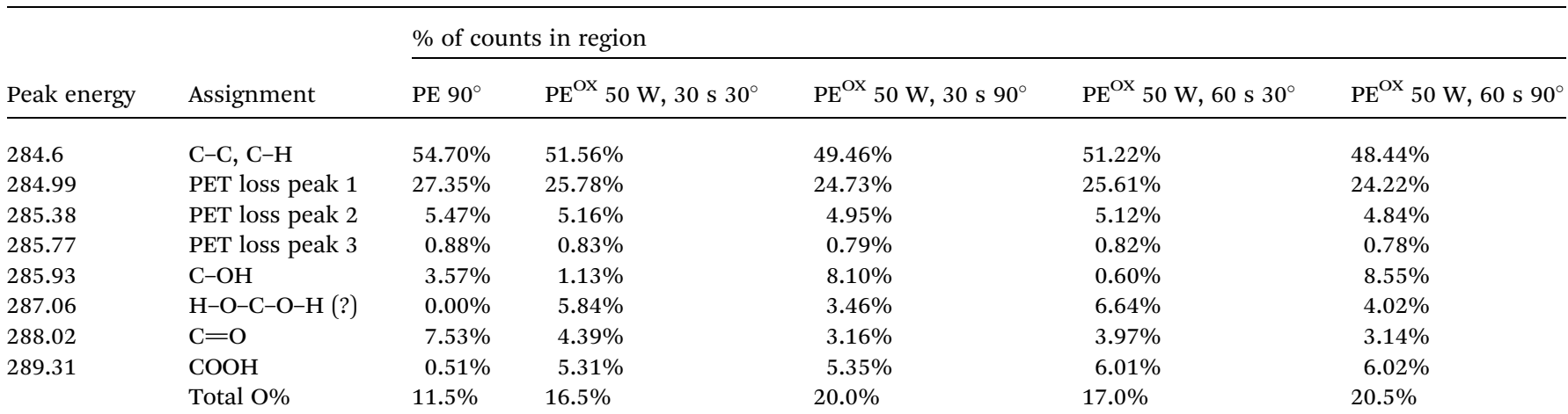

The XPS data for oxygen functional groups on $\mathrm{PE}$ and $\mathrm{PE}^{\mathrm{OX}}$ are summarised in Table 1 . The $30^{\circ}$ data are indicative of surface groups as the beam penetrates less than in the $90^{\circ}$ configuration. The treated samples show a slight enhancement over time in carbonyls relative to $\mathrm{OH}$, consistent with oxidation proceeding from alcohol, to aldehyde, to carboxylic acid. The difference as a function of angle of incidence suggests that the surface is slightly enriched with carbonyls (relative to $\mathrm{OH}$ ) compared to the depth of the film.

The XRD pattern of non-stretched PE (prestretched by the manufacturer during production) and $2 \times$ stretched (using the LD stretcher) film are shown in Fig. 3. Our X-ray diffraction studies seem to support the conclusion that stretching increases the crystallinity of PE. The two reflections at $22^{\circ}$ and $24^{\circ}$ are characteristic of the typical orthorhombic Pnam crystalline phase of PE. ${ }^{23}$ The intensity of these reflections, however, suggests that they are not the prevalent phase in the unstretched film. The intense reflections at $14^{\circ}$ and $17^{\circ}$ dominate the diffractogram. There is an excellent agreement between these and other less intense reflections and the monoclinic $C c$ phase of polyisopropylene (PP), ${ }^{24}$ suggesting that the film used is in fact a ethylene/propylene co-polymer-with the propylene component being highly crystalline. This is further supported by the NMR analysis (see ESI $\dagger$ ) which suggests that $1.5 \%$ of the film by weight is PP. There are also several less intense reflections of varying sharpness present in the diffractogram, suggesting presence of at least one more crystalline or semicrystalline phase in the sample.

There are significant changes in the diffractogram after stretching $2 \times$. The relative intensities of the reflections characteristic of the orthorhombic crystallites are much stronger than for an unstretched film, suggesting that the crystallites of this structure are now prevalent in the film. The relative intensities of the $22^{\circ}$ and $24^{\circ}$ reflections (orthorhombic PE) have also

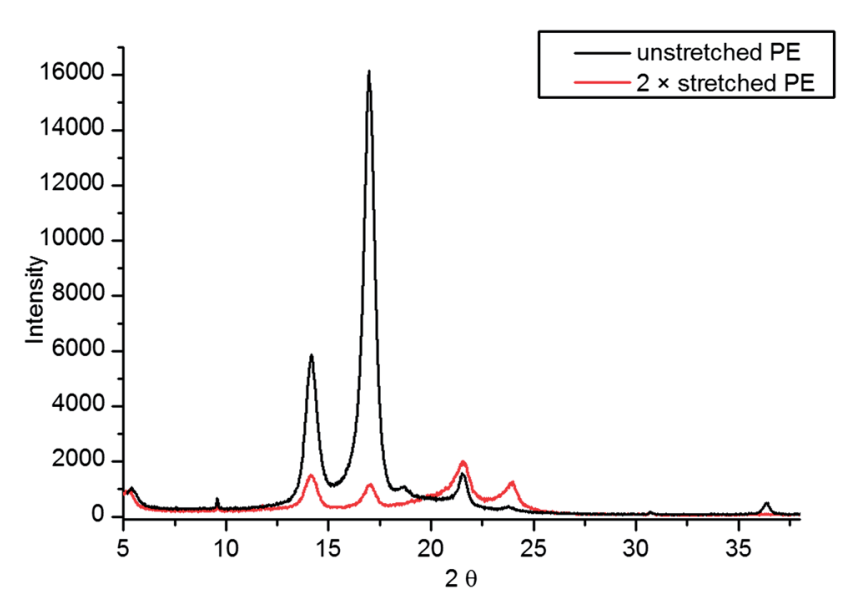

Fig. 3 X-ray diffraction patterns of un-stretched and $2 \times$ stretched PE films. 
changed after stretching suggesting a change in preferred orientation of the crystallites in the film. Other differences include appearance of new reflections at $19^{\circ}$ and $36^{\circ}$ as well as disappearance of a low intensity sharp reflection at $12^{\circ}$, which suggests profound changes in the relative amounts and orientations of different crystal phases present throughout the film.

Using the Scherrer equation it was possible to estimate the size of the crystallites in the polymer. ${ }^{25}$ The estimated values for PP crystallites do not change after stretching, while the average size of the PE crystallites decreases with stretching, which is consistent with creation of new (smaller) crystallites in the polymer.

\section{Orienting small molecules on PE}

Progesterone: a medium polarity molecule that orients in the same manner on $\mathrm{PE}$ and $\mathrm{PE}^{\mathrm{Ox}}$. Fig. 4 shows the LD spectra on both $\mathrm{PE}$ and $\mathrm{PE}^{\mathrm{OX}}$ films of a medium polarity molecule, progesterone, with a single $\pi-\pi^{*}$ transition in the accessible region of the spectrum. The progesterone spreads somewhat more uniformly on $\mathrm{PE}^{\mathrm{OX}}$ when dissolved in methanol than on PE when dissolved in chloroform which seems to have resulted in slightly better (in terms of $\mathrm{LD}^{\mathrm{r}}$ ) spectra and higher orientation factor $S$. The $\mathrm{LD}^{\mathrm{r}}$ traces are fairly flat as expected for a single transition. If we assume that (i) the molecular orientation is uniaxial (see below for more discussion of this); (ii) the long axis is the $z$-axis, and (iii) the transition is polarized along the carbonyl bond, then $S \sim 0.12$ for $1.8 \times$ stretch $\mathrm{PE}$ and 0.13 for $1.8 \times$ stretch $\mathrm{PE}^{\mathrm{OX}}$. We can therefore conclude that $\mathrm{PE}^{\mathrm{OX}}$ orients progesterone in much the same way as PE but with marginally higher efficiently and uniformity.
We wished to optimise the sample orientation in the films and also to check whether degree of stretching affected the spectroscopy. The progesterone spectra suggest that the maximum LD is obtained with a stretch factor of $\sim 1.8$ and a maximum $\mathrm{LD}^{\mathrm{r}}$ with factors of $\sim 2.0$. This ran counter to our expectation that more stretching would always correspond to more orientation. For progesterone, and for the other analytes discussed below, the variation in $\mathrm{LD}^{\mathrm{r}}$ maximum proved to be due mainly to variations in the absorbance; molecules unoriented on the film absorb light but may not contribute to the LD. The film thickness did not change significantly with stretching, though the changes in the spectra do show changes in the binding of the analytes to the PE.

Uniaxial orientation. The analysis of LD is facilitated if we can use eqn (2), which holds for uniaxially oriented samples. We have concluded (see ESI $\dagger$ ) that a sample is uniaxially oriented if its LD signal depends on the rotation about the $X$-axis (direction of propagation of light) according to

$$
\begin{aligned}
\mathrm{LD}^{\mathrm{r}} & =\frac{\mathrm{LD}}{A_{\text {iso }}}=\frac{A_{Z}-A_{Y}}{A_{\text {iso }}} \\
& =\frac{3}{4}\left(3\left\langle\cos ^{2} \theta\right\rangle-1\right)\left(3\left\langle\cos ^{2} \alpha\right\rangle-1\right) \cos (2 \chi) \\
& =\frac{3}{2} S\left(3\left\langle\cos ^{2} \alpha\right\rangle-1\right) \cos (2 \chi)
\end{aligned}
$$

where $\chi$ is the angle between $Z$ (the stretch direction of the film) and the horizontal direction, $\theta$ is the angle between $z$ (the molecular orientation axis) and $Z$, and $A_{\text {iso }}$ is the isotropic absorbance. If there are multiple populations of molecules or less symmetric orientation mechanisms, then a different function of

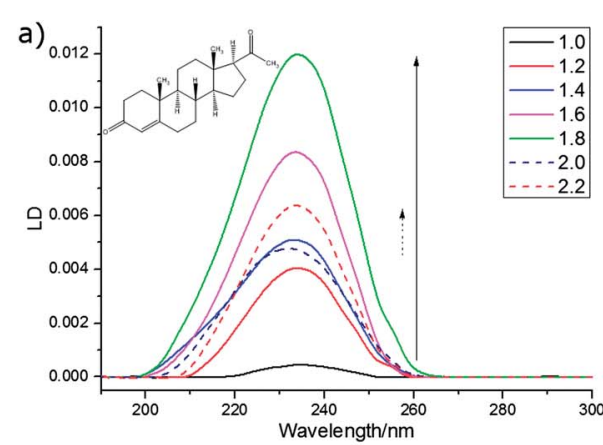

c)

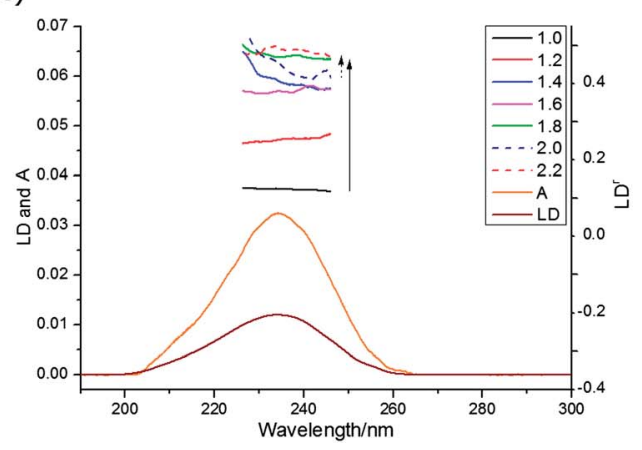

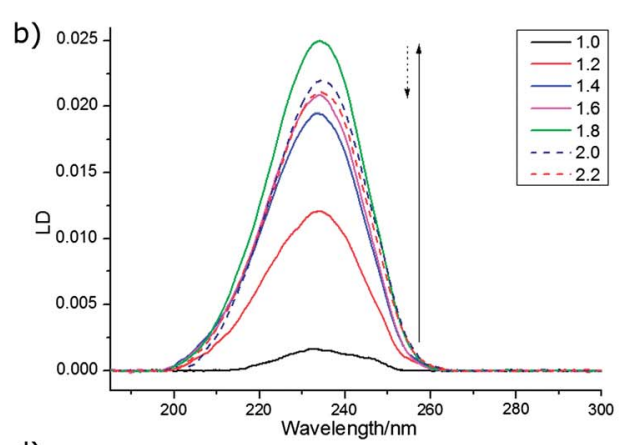

d)

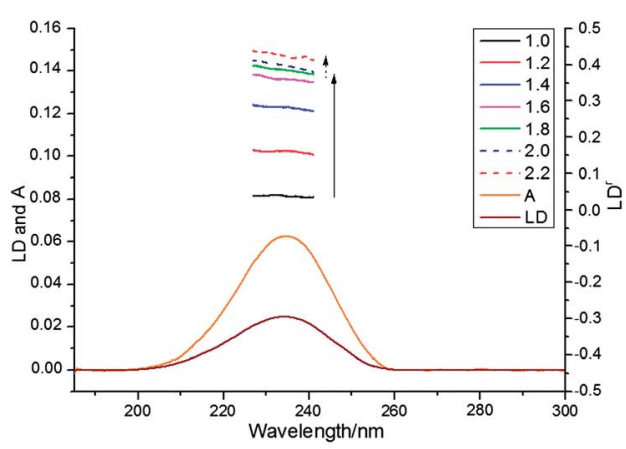

Fig. 4 Spectra of progesterone (deposited from $0.65 \mathrm{mg} \mathrm{mL}^{-1}$ in chloroform for PE and methanol for PE ${ }^{\mathrm{OX}}$ and left to dry). LD on (a) PE, and (b) $P E^{O X}$ prestretched by factors ranging from 1 to $2.2 \times$. (c) and (d) LD for data from (a) and (b) respectively overlaid with absorbance and LD spectra for $1.8 \times$ stretch. 

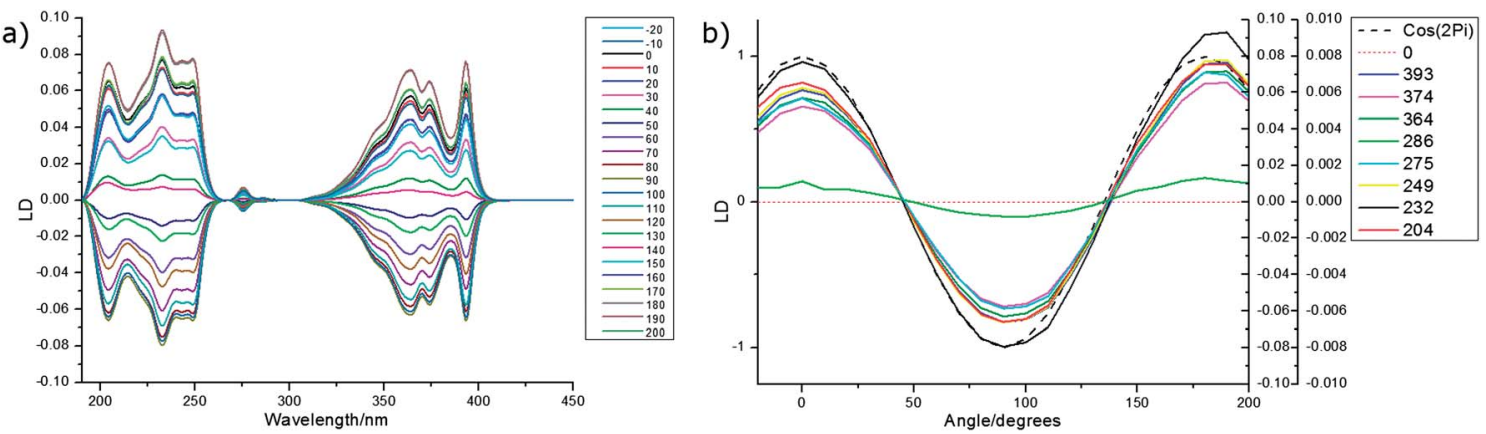

Fig. 5 (a) LD (not baseline corrected) of pyrenecarboxaldehyde (deposited from $0.35 \mathrm{mg} \mathrm{mL}^{-1}$ in methanol) on $\mathrm{PE}{ }^{\mathrm{OX}}$ stretched $1.8 \times$ as a function of rotation angle, $\chi$, of the film. $0^{\circ}$ is where the stretch direction is as close to horizontal as loading the film in a small circle enabled. (b) LD at wavelength indicated as a function of $\chi$. Dashed line is the plot of $\cos (2 \chi)$. See Fig. 7 for molecular structure.

a)

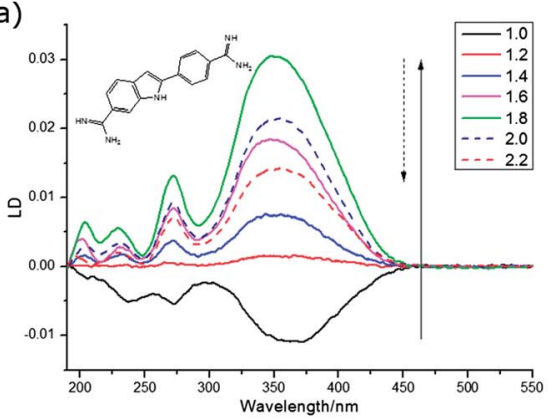

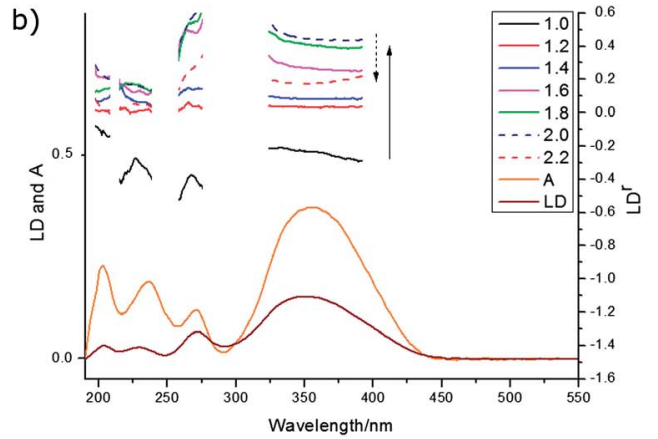

Fig. 6 Spectra of DAPI on $\mathrm{PE}^{\mathrm{OX}}$ (deposited from $0.4 \mathrm{mg} \mathrm{mL}^{-1}$ in methanol and left to dry). (a) LD prestretched by factors ranging from 1 to $2.2 \times$. (b) LD for data from (a) overlaid with absorbance and LD spectra for $1.8 \times$ stretch.

$\chi, \alpha$, and $\theta$ is observed. Thus, we can use plots of LD as a function of $\chi$ to determine whether a molecule is uniaxially oriented.

An overlay of the LD (not baseline corrected) of pyrenecarboxaldehyde (PyCO) as a function of film-rotation angle, $\chi$, is shown in Fig. 5 along with a plot of signals at the LD maxima as a function of $\chi$. The $\cos (2 \chi)$ dependence of the signals means that either the molecule behaves as a uniaxial rod or the film ensures uniaxial orientation or a combination of the two. The equivalent plots for the other molecules discussed below are given in $\mathrm{ESI} \dagger$ for both $\mathrm{PE}$ and $\mathrm{PE}^{\mathrm{OX}}$ (Fig. SI1-SI4, ESI $\dagger$ ). Within experimental error of loading the sample and noting none of the spectra are baseline corrected, each molecule and film studied in this work behave consistently with a uniaxial orientation mechanism.

DAPI. DAPI (4',6-diamidino-2-phenylindole) is a good test case for $\mathrm{PE}^{\mathrm{OX}}$ as DAPI fails to orient on PE and there is literature PVA film data available for comparison. ${ }^{26}$ Fig. 6 shows the variable stretch LD spectra of DAPI on $\mathrm{PE}^{\mathrm{OX}}$. Our data go to lower wavelength than the PVA spectrum but in other respects are similar. The broad band at approximately $350 \mathrm{~nm}$ actually consists of two transitions, one at $330 \mathrm{~nm}$ and another at $380 \mathrm{~nm}$. Analysis of their DAPI PVA data led Kubista et al. to conclude that $S=0.66$ and that the $380 \mathrm{~nm}$ transition was the most oriented and was polarized close to the indole-phenyl bond. ${ }^{26}$ They assigned polarizations to the other transitions but were not able to determine whether they were $+d^{\circ}$ or $-d^{\circ}$ from $z$.
Comparison of their data with our $\mathrm{PE}^{\mathrm{OX}}$ data (Fig. 6) give us a way to determine this since the relative magnitudes of peaks on $\mathrm{PVA}$ and $\mathrm{PE}^{\mathrm{OX}}$ differ, indicating a slightly different orientation.

Table 2 Angles between DAPI transition moments and the orientation axis in the PVA data of Kubista et al. ${ }^{26}$ and the PE ${ }^{\mathrm{OX}}$ data of this work. $P E^{O X} L^{r}$ data are for $1.8 \times$ stretch. The directions illustrated in Fig. 7 are consistent with both data sets

\begin{tabular}{llll}
\hline Wavelength & PVA angle & $\mathrm{PE}^{\mathrm{OX}}$ angle & $\mathrm{LD}^{\mathrm{r}}$ on $\mathrm{PE}^{\mathrm{OX}}$ \\
\hline $380 \mathrm{~nm}$ & $0-5^{\circ}$ & $26^{\circ}$ & 0.39 \\
$330 \mathrm{~nm}$ & $15-17^{\circ}$ & $17^{\circ}$ & 0.48 \\
$270 \mathrm{~nm}$ & $12-19^{\circ}$ & $0^{\circ}$ & 0.55 \\
$228 \mathrm{~nm}$ & $>50^{\circ}$ & $45^{\circ}$ & 0.14
\end{tabular}

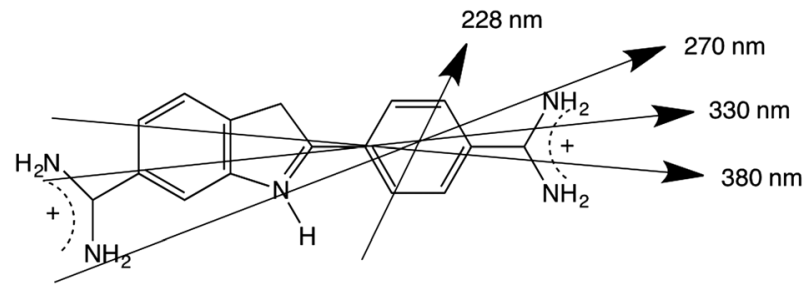

Fig. 7 4',6-Diamidino-2-phenylindole illustrating approximate transition polarizations consistent with this work and the PVA data of ref. 26. 

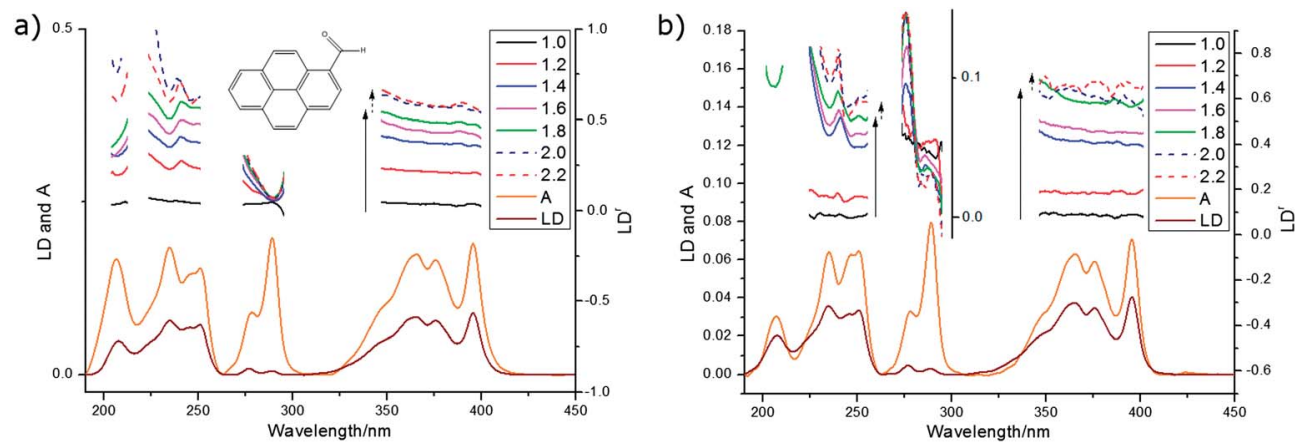

Fig. $8 \mathrm{LD}^{r}$ spectra of pyrenecarboxaldehyde (deposited from $0.4 \mathrm{mg} \mathrm{mL}^{-1}$ in methanol and left to dry) on films prestretched by factors ranging from 1 to $2.2 \times$ overlaid with absorbance and LD spectra for $1.8 \times$ stretch. (a) PE and (b) $\mathrm{PE}^{\mathrm{OX}}$.

The $270 \mathrm{~nm}$ transition for $\mathrm{PE}^{\mathrm{OX}}$ (Table 2) has the largest value of $\mathrm{LD}^{\mathrm{r}}$, so we assume it is along $z$. It then follows that for the $1.8 \times$ stretch $\mathrm{PE}^{\mathrm{OX}}$ experiment, $S=0.18$. The directions of $z$ and the other transition moments can then be deduced from $\mathrm{LD}^{\mathrm{r}}$ data and are given in Table 2 where the PVA results are also summarized. Transition moment polarizations consistent with both data sets are illustrated in Fig. 7. Overall, the quality of the DAPI-PE ${ }^{\text {OX }}$ spectra suggest $\mathrm{PE}^{\mathrm{OX}}$ can replace PVA for polar molecules making a much simpler quicker experiment.

1-Pyrenecarboxaldehyde: different binding to $\mathbf{P E}$ and $\mathbf{P E}^{\mathrm{OX}}$. 1-Pyrenecarboxaldehyde (PyCO, Fig. 8) provides an interesting illustration of how $\mathrm{PE}$ and $\mathrm{PE}^{\mathrm{OX}}$ may, in some cases, be used to provide complementary data that can be used in a manner similar to that done for DAPI and $\mathrm{PE}^{\mathrm{OX}}$ above since PyCO turns out to orient slightly differently on the two films. The aldehyde group of PyCO is polar and the pyrene is very non-polar. LD data for the pyrene chromophore on films and in liposomes are available in the literature ${ }^{7,8,16}$ (see Table 3). The absorbance spectroscopy of PyCO (Fig. 8 and SI3, ESI $\dagger$ ) is similar to that of pyrene but shifted to longer wavelengths with additional features including the $234 \mathrm{~nm}$ peak. PyCO has $C_{\mathrm{s}}$ symmetry (pyrene is $D_{2 \mathrm{~h}}$ ), so its $\pi-\pi^{*}$ transition are constrained to be in the plane but not along either the long axis or the short axis of the pyrene chromophore. The $\mathrm{LD}$ and $\mathrm{LD}^{\mathrm{r}}$ spectra (Fig. 8, only one LD spectrum is shown as they retain the same shape with stretching Fig. SI5, ESI $\dagger$ ) indicate spectral ranges of single polarizations as summarised in Table 3 . With $\mathrm{PE}^{\mathrm{OX}}$, the $207 \mathrm{~nm}$ transition is closest to $z$ (though its $\mathrm{LD}^{\mathrm{r}}$ value does depend on how the baseline correction is done) then the $350-400 \mathrm{~nm}$ band,

Table $3 L^{r}$ data for 1-pyrenecarboxaldehyde on $\mathrm{PE}$ and $\mathrm{PE} \mathrm{OX}^{\mathrm{X}}$ at $1.8 \times$ stretch, literature transition polarizations for pyrene, and PyCO transition moment polarizations $\left( \pm 3^{\circ}\right)$ with respect to the $\mathrm{PE}^{\mathrm{OX}} z$ as illustrated in Fig. 6 (+ denotes anticlockwise rotation as illustrated)

\begin{tabular}{lllll}
\hline Wavelength & $\begin{array}{l}\mathrm{LD}^{\mathrm{r}} \\
\text { on PE }\end{array}$ & $\begin{array}{l}\mathrm{LD}^{\mathrm{r}} \text { on } \\
\mathrm{PE}^{\text {Ox }}\end{array}$ & $\begin{array}{l}\text { Pyrene } \\
\text { assignment }\end{array}$ & $\begin{array}{l}\text { PyCO } \\
\text { assignment }\end{array}$ \\
\hline $207 \mathrm{~nm}$ & 0.39 & 0.64 & & $\sim 0^{\circ}$ \\
$234 \mathrm{~nm}$ & 0.53 & 0.62 & & $+9^{\circ}$ \\
$246-253 \mathrm{~nm}$ & 0.57 & 0.51 & $220-240 \mathrm{~nm}$ : long & $-21^{\circ}$ \\
$276 \mathrm{~nm}$ & 0.28 & 0.13 & $260 \mathrm{~nm}$ : long & $-46^{\circ}$ \\
$288 \mathrm{~nm}$ & 0.077 & 0.038 & $270-280 \mathrm{~nm}$ : short & $+53^{\circ}$ \\
$350-400 \mathrm{~nm}$ & 0.51 & 0.56 & $300-340 \mathrm{~nm}$ : long & $-16^{\circ}$
\end{tabular}

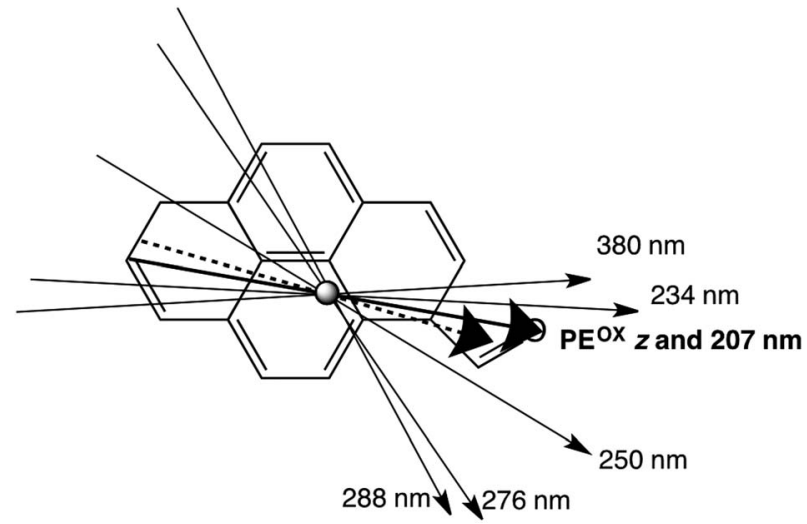

Fig. 9 Polarizations of PyCO transitions assuming the orientation direction on $\mathrm{PE}^{\mathrm{OX}}$ is as indicated by the thick solid line (the longest molecular axis with $O$ lying flat on the film). The thick dashed line is then the orientation axis on PE.

whereas with $\mathrm{PE}, 246-253 \mathrm{~nm}$ has the largest $\mathrm{LD}^{\mathrm{r}}$, so is closest to $z$. In pyrene these transitions are all long-axis polarized. We speculate that the two films orient PyCO differently because the carbonyl interacts with the more polar $\mathrm{PE}^{\mathrm{OX}}$ surface but not with PE. The solid arrow in Fig. 9 is thus a reasonable estimate of $z$ for $\mathrm{PE}^{\mathrm{OX}}$ with (the lower limit of) $S=0.21$. An iterative trial and error process led us to conclude that $S$ was the same for both films; there was $6^{\circ}$ difference between their molecular orientation axes (the orientation axis on PE is illustrated by the dashed line in Fig. 9 and by the solid arrow on $\mathrm{PE}^{\mathrm{OX}}$ ); and the transition polarizations are approximately as illustrated in Fig. 9 and summarized in Table 3. The $207 \mathrm{~nm}, 380 \mathrm{~nm}$ and $234 \mathrm{~nm}$ transitions are thus close to the pyrene long axis, whereas the others, particularly the former pyrene short axis polarized transitions, are significantly perturbed from their pyrene values.

Anthracene. Anthracene is a well-studied molecule with extensive literature on its spectroscopy in solution, crystal and on films. We begin our analysis by accepting the work of Friedrich et al. ${ }^{27}$ namely that the long wavelength band for anthracene is the ${ }^{1} \mathrm{~L}_{\mathrm{a}}$ band of $B_{2 \mathrm{u}}$ symmetry (i.e. $y$-polarized in an axis system where $z$ is the anthracene long axis and $y$ is its in-plane short axis) with the origin at about $379 \mathrm{~nm}$ (depending on the solvent). This transition has clear vibronic 

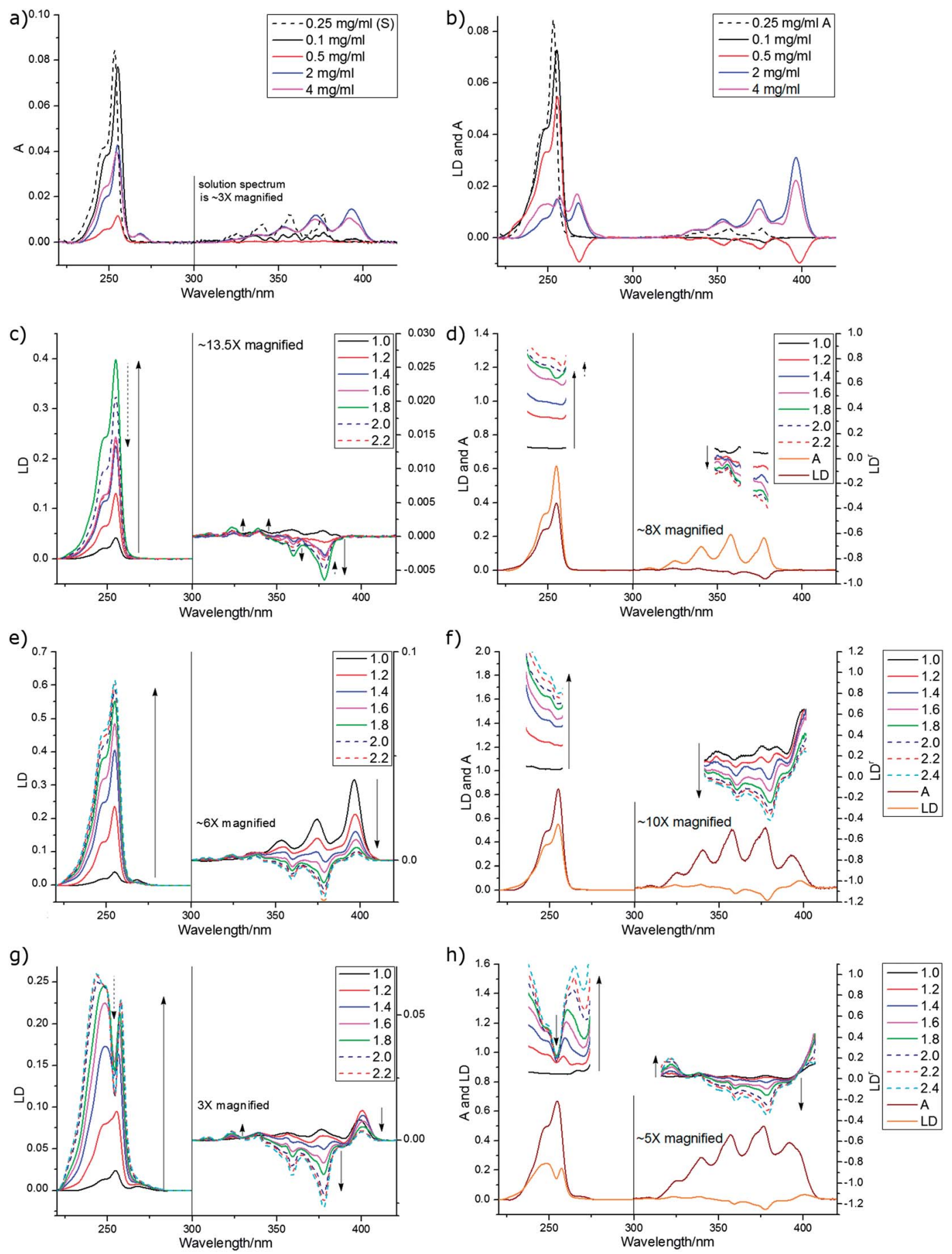

Fig. 10 Spectra of anthracene. (a) Absorbance of anthracene in solution ( $0.3 \mathrm{mg} \mathrm{mL}^{-1}$ cyclohexane) overlaid on absorbance spectra of $1.8 \times$ stretch films (PE) deposited from chloroform solutions of the concentrations indicated in the figure. (b) LD corresponding to (a) film spectra with solution absorbance overlaid normalised to have areas under the curves equal to one. (c) LD (deposited from $0.1 \mathrm{mg} \mathrm{mL}^{-1}$ solution in methanol, to ensure monomer, see text) on $\mathrm{PE}^{\mathrm{OX}}$ as a function of stretch factor (indicated - sample deposited after stretching). LD deposited from solutions in chloroform in (e) $1 \mathrm{mg} \mathrm{mL}^{-1}$ (g) $8 \mathrm{mg} \mathrm{mL}^{-1}$ as a function of stretch (sample added before stretching). (d), (f), and (h) LD' for data from respectively (c), (e), and (g) overlaid with absorbance and LD spectra for $1.8 \times$ stretch.

progressions in solution and on film (Fig. 10a). We can see totally symmetric $\left(a_{1 \mathrm{~g}}\right)$ vibrational progressions due to a $\sim 1420 \mathrm{~cm}^{-1}$ stretching vibration and a $399 \mathrm{~cm}^{-1}$ skeletal bending vibration (which is apparent as a high energy shoulder at $\sim 372 \mathrm{~nm}$ in our spectra). Overlaid on these is a $1480 \mathrm{~cm}^{-1}$
(Michl et al. ${ }^{28}$ ) or $1630 \mathrm{~cm}^{-1}$ or perhaps $1481 \mathrm{~cm}^{-1}$ (Friedrich et $a{ }^{27}{ }^{27} b_{3 \mathrm{~g}}$ progression that mixes in intensity from the $250 \mathrm{~nm}$ ${ }^{1} B_{\mathrm{b}}$ long axis polarized band resulting in intensity of $B_{1 \mathrm{u}}$ symmetry. The electronic polarization of this progression is therefore along $z$. 
Table 4 Approximate effective polarizations (error is of the order of 5\%) of the vibronic components of the $379 \mathrm{~nm} B_{2 \mathrm{u}}$ vibronic progression of anthracene, and the fractions of short axis and long axis electronic contributions to the total determined from $1.8 \times$ stretch data in Fig. $10 \mathrm{~b}$ assuming the $254 \mathrm{~nm}$ band is long axis polarised. Calculations are performed on the centre of each band from low energy half height to high energy half height. The fraction of the $a_{\mathrm{g}}$ progression is fraction of intensity that is due to the short axis polarized component assuming the same oscillator strength for the two components

\begin{tabular}{lllll}
\hline $\begin{array}{l}\text { Wavelength maximum assuming } \\
1400 \mathrm{~cm}^{-1} \text { progression }\end{array}$ & $\begin{array}{l}\text { Wavelength maximum } \\
\text { of LD }\end{array}$ & LD sign & $\alpha_{\text {effective }}$ & $\begin{array}{l}\text { Fraction of } a_{\mathrm{g}} \text { progression } \\
\text { (assuming only monomer present) }\end{array}$ \\
\hline $378 \mathrm{~nm}$ & $378 \mathrm{~nm}$ & - & $75^{\circ}-90^{\circ}$ & $0.93-1.0$ \\
$360 \mathrm{~nm}$ & $360 \mathrm{~nm}$ & - & $62^{\circ}$ & 0.78 \\
$342 \mathrm{~nm}$ & $339 \mathrm{~nm}$ & + & $47^{\circ}$ & 0.59 \\
$327 \mathrm{~nm}$ & $323.5 \mathrm{~nm}$ & + & $34^{\circ}$ & 0.45 \\
$313 \mathrm{~nm}$ & $309 \mathrm{~nm}$ & + & $31^{\circ}$ & 0.36
\end{tabular}

A further complication is the possibility of anthracene forming dimers (or other higher order structures).$^{29}$ Chandross et al. identified two dimer forms with different spectroscopy: a so-called sandwich dimer which required a cryogenic glass to stabilize it and a stable dimer (or higher order structure) which they concluded had $\sim 55^{\circ}$ between the short axes and not more than $5^{\circ}$ between the long axes. They were unable to study the $254 \mathrm{~nm}$ band of their stable dimer of anthracene as they found that the transition was shifted beyond their range of observation. ${ }^{29}$ Consistent with these structures, the crystal structure ${ }^{30}$ has two distinct anthracene pairs, one parallel and slightly displaced and one skewed with angles of $54^{\circ}$ between the short axes and $14^{\circ}$ between the long axes (more information on the angles between the monomer units is given in Table SI1, ESI $\dagger$ ). However, we found we could not interpret our film data in terms only of these structures.

Despite being a non-polar molecule, anthracene orients well on both $\mathrm{PE}$ and $\mathrm{PE}^{\mathrm{OX}}$. The $\mathrm{PE}^{\mathrm{OX}}$ data are usually slightly better as the sample spreads more uniformly on the film. LD filmrotation experiments show that the anthracene orientation is always uniaxial in our experiments (Fig. SI4, ESI $\dagger$ ). By depositing anthracene from a dilute solution of $\mathrm{CHCl}_{3}\left(0.1 \mathrm{mg} \mathrm{mL} \mathrm{mL}^{-1}\right)$ we are able to ensure a fairly pure monomer spectrum as shown by a comparison of the film and solution (methylcyclohexane) absorption spectra (Fig. 10a) where the solution has a slight blue shift relative to the $0.1 \mathrm{mg} \mathrm{mL}^{-1}$ film spectra. The monomer spectra as a function of stretch factor are shown in Fig. $10 \mathrm{c}$ and $\mathrm{d}$. The longest wavelength band (the $0-0$ vibronic band of the ${ }^{1} \mathrm{~L}_{\mathrm{a}}$ band) in solution absorbance, film absorbance and film LD occurs at $\sim 378 \mathrm{~nm}$ and the main vibronic components occur at $\sim 1400 \mathrm{~cm}^{-1}$ intervals. The third vibronic band has a positive LD signal indicating that the contribution of the $254 \mathrm{~nm}$ band is becoming dominant. If we assume that the LD from 245-258 $\mathrm{nm}$ is long axis polarized with $\alpha=0^{\circ}$, then from eqn (2) $S \sim 0.22$ for the $1.8 \times$ stretch ( $\mathrm{PE}$ or $\mathrm{PE}^{\mathrm{OX}}$ ). It then follows that the vibronic components of the $377 \mathrm{~nm}$ band have average angles as indicated in Table 4 with gradually increasing contribution from the long axis polarized electronic transition. The fact that the $378 \mathrm{~nm}$ (fundamental band) $\alpha$ is not $90^{\circ}$ reflects the fact that we do have some oligomeric species present (see below).

Anthracene dimers or higher order structures. If we prepare anthracene films from higher concentration solutions we observe absorbance and LD signals in the region of $400 \mathrm{~nm}$ and see intensity at $\sim 268 \mathrm{~nm}$ and changes in the $245 \mathrm{~nm}$ region. What is observed depends on the way the experiment is done, whether the films are pre-stretched or stretched after the sample is added and also on the concentration of the solution that is added. Some examples are given in Fig. 10a, b, e-h showing the effect of exciton coupling ${ }^{31}$ between anthracene units with intensity appearing at either high energy or low energy or both (see ESI† for more details).

Table 5 Different component spectra identified in the anthracene film LD spectra of Fig. 10. 'Large' indicates where the long wavelength region has enhanced intensity relative to the $250 \mathrm{~nm}$ region. Vibronic progressions are indicated where they are apparent. Sh denotes shoulder

\begin{tabular}{|c|c|c|c|c|c|c|}
\hline $\begin{array}{l}\text { Spectrum in which } \\
\text { component is most apparent }\end{array}$ & $\begin{array}{l}\text { LD at } \\
\sim 230 \mathrm{~nm}\end{array}$ & $\begin{array}{l}\text { LD at } \\
\sim 254 \mathrm{~nm}\end{array}$ & $\begin{array}{l}\text { LD at } \\
\sim 268 \mathrm{~nm}\end{array}$ & $\mathrm{LD}$ at $379 \mathrm{~nm}$ & $\begin{array}{l}\text { LD at } \\
\sim 400 \mathrm{~nm}\end{array}$ & $\begin{array}{l}\text { Geometry } \\
\text { consistent with LD }\end{array}$ \\
\hline Monomer & 0 & +ve & 0 & $\begin{array}{l}\text {-ve, from } 379 \mathrm{~nm} \\
14000 \mathrm{~cm}^{-1}\end{array}$ & 0 & Fig. 10a \\
\hline $0.5 \mathrm{mg} \mathrm{mL}^{-1}$, Fig. $10 \mathrm{~b}$ & +ve (sh) & $(?)$ & - ve & $\begin{array}{l}\text {-ve, from } 399 \mathrm{~nm}, \\
16000 \mathrm{~cm}^{-1} \text { (large) }\end{array}$ & & Fig. 10d \\
\hline $\begin{array}{l}1 \mathrm{mg} \mathrm{mL} \mathrm{m}^{-1} \text {, Fig. 10e, } \\
\text { unstretched }\end{array}$ & 0 & $(?)$ & +ve & $\begin{array}{l}\text { +ve, from } 396 \mathrm{~nm}, \\
14800 \mathrm{~cm}^{-1} \text { (large) }\end{array}$ & & Fig. 10c \\
\hline $\begin{array}{l}8 \mathrm{mg} \mathrm{mL}{ }^{-1} \text {, Fig. } 10 \mathrm{~g} \\
\text { stretched }\end{array}$ & 0 & $-\mathrm{ve}$ & 0 & $\begin{array}{l}\text {-ve (large), from } 378 \mathrm{~nm} \text {, } \\
\sim 14000 \mathrm{~cm}^{-1}\end{array}$ & 0 & Fig. 10b \\
\hline
\end{tabular}


Most of our concentrated films showed positive LD signals for the longest wavelength signal at $397 \mathrm{~nm}$ (the maximum of the stable dimer of Chandross et al. ${ }^{29}$ ) and at 266-267 $\mathrm{nm}$. Some showed negative signals at both $399 \mathrm{~nm}$ and at $268 \mathrm{~nm}$. There are at least 4 different component spectra apparent in our spectra, all overlaid on each other and the monomer. The monomer is dominant at low concentration (see above) and becomes dominant at high stretch with medium concentration stock solutions $\left(1 \mathrm{mg} \mathrm{mL} \mathrm{mL}^{-1}\right.$ ), reflecting the increased crystallinity of the PE with stretch making more monomer binding sites available. The high concentration $\left(8 \mathrm{mg} \mathrm{mL}{ }^{-1}\right)$ spectra seems to be an overlay of the $1 \mathrm{mg} \mathrm{mL}^{-1}$ unstretched spectrum with one that has negative LD occurring at the monomer transition energies for both the long and short axis polarized transitions. There is also a spectrum that has positive signals at energies lower than the monomer transitions (396 $\mathrm{nm}$ plus vibronic progression and $268 \mathrm{~nm}$ ), but which matching higher energy exciton component is small or zero. Another spectrum with negative LD at $399 \mathrm{~nm}$ and $268 \mathrm{~nm}$ and positive (shoulder) at $230 \mathrm{~nm}$ is observed in a $0.5 \mathrm{mg} \mathrm{mL}{ }^{-1}$ film. The features of these spectra are summarized in Table 5.

The monomer, as discussed above, is uniaxially oriented with its long axis along the stretch direction giving positive $250 \mathrm{~nm} \mathrm{LD}$ and negative $379 \mathrm{~nm}$ LD. The complex spectral features discussed above are the result of higher order anthracene structures adopting other orientations on the film and exciton coupling to give signals at higher and lower energies usually with one positive and one negative in sign.

To have completely negative LD at the monomer transitions ( $8 \mathrm{mg} \mathrm{mL} \mathrm{m}^{-1}$, last line of Table 5), the anthracenes must be oriented with their planes perpendicular to the stretch direction. If the anthracenes have parallel molecular planes, but the nearest neighbor long axes are twisted by $90^{\circ}$ with respect to one another then there is zero exciton energy shift (see ESI $\dagger$ ). An extended cross-hatched structure as illustrated in Fig. 11b is consistent with this and is expected to be stable on the stretched film. We postulate this is what is happening in the $8 \mathrm{mg} \mathrm{mL}^{-1}$ film. The geometries giving rise to the $1 \mathrm{mg} \mathrm{mL}^{-1}$ unstretched and $0.5 \mathrm{mg} \mathrm{mL} \mathrm{mL}^{-1}$ spectra are harder to identify uniquely. Possible arrangements include the following. A geometry similar to that of the skew pair in the crystal structure ${ }^{32}$ gives rise to almost no intensity for the out-of-phase couplings for both long and short-axis anthracene transitions and (if the long axes are along the stretch direction) positive LD for the in-phase

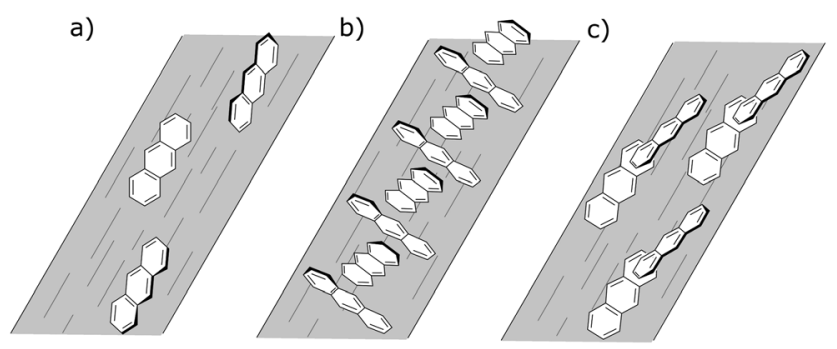

Fig. 11 The three proposed orientations of anthracene on $\mathrm{PE}^{\mathrm{OX}}$ films, (a) uniaxially oriented single molecules, (b) extended cross-hatched structure and (c) skewed dimers. coupling (Table SI2, ESI $\dagger$ ). A geometry where one layer of anthracenes are aligned in a head-to-tail trail along the stretch direction (as are the monomers) and a second layer is tilted as in the crystal $\left(14.4^{\circ}\right.$ between long axes and $54^{\circ}$ between short axes) but displaced enough to ensure $V<0$ (Table SI1, ESI $\dagger$ ) as illustrated in Fig. 11c accounts for the $1 \mathrm{mg} \mathrm{mL}^{-1}$ unstretched spectrum. The $0.5 \mathrm{mg} \mathrm{mL}{ }^{-1}$ spectrum is consistent with a geometry where the first layer is the same as this but the second layer has the molecules parallel to the first and rotated about $X$ axis by $2 \gamma=60^{\circ}$ (Fig. 11c). This geometry has $V>0$ (Table SI1, ESI $\dagger$ ), both in-phase and out-of-phase short axis transitions positive, and long-axis in-phase positive but out-of-phase negative.

\section{Conclusions}

In this work we have shown that we can change the surface properties of polyethylene (PE) by oxygen plasma treatment enough to enable even the most polar of small molecules to be oriented on its oxidised surface ( $\left.\mathrm{PE}^{\mathrm{OX}}\right)$. Overall, the quality of the DAPI $\mathrm{PE}^{\mathrm{OX}}$ spectra suggest $\mathrm{PE}^{\mathrm{OX}}$ can replace polyvinyl alcohol for polar molecules making a much simpler and quicker experiment. In general we found that the sample deposition was more uniform with the $\mathrm{PE}^{\mathrm{OX}}$ surfaces making it usually the preferred substrate. The orientation of our films increased with stretch factor due to changes in crystallinity and crystallite orientation in the PE substrate (which also includes crystalline polypropylene). For some molecules (e.g. 1-pyrenecarboxaldehyde) we found the different interactions between analyte and the two films enabled us to assign transition moments of low symmetry molecules. Easy transition moment assignment follows if the molecules orient uniaxially on the polymer film. We have shown that a simple matrix rotation experiment can be used to determine whether or not the orientation may be assumed to be uniaxial.

Our work on anthracene stretched film samples shows the importance of knowing what is being studied. For anthracene, the analyte must be deposited from quite low concentration solutions to ensure monomers dominate the spectroscopy. Films prepared from high concentration stock solutions of anthracene showed a variety of different higher order structures with corresponding variations in spectra resulting from different exciton couplings. The LD sign patterns can be readily used to eliminate possible oligomer geometries, however, the overlay of spectra for different structures usually makes it challenging to definitively identify structures.

\section{Acknowledgements}

XPS data were collected at the Science City Photoemission Facility, University of Warwick and contact angle measurements were performed using the Science City Advanced materials 2 Facility. The equipment used was obtained through Birmingham Science City: Innovative Uses for Advanced Materials in the Modern World with support from Advantage West Midlands (AWM) and part funded by the European Regional Development Fund (ERDF). The Jasco J-815 spectropolarimeter was obtained 
through Biotechnology and Biological Sciences Research Council grant (BBSRC; grant no. BB/F011199/1). The authors would like to thank Richard Walton for his help with X-ray diffraction experiments and Christopher Kay for his help with NMR data collection and analysis.

\section{Notes and references}

1 B. Nordén and F. Tjerneld, FEBS Lett., 1976, 67, 368-370.

2 P. E. Schipper, B. Nordén and F. Tjerneld, Chem. Phys. Lett., 1980, 70, 17-21.

3 C. Hiort, B. Nordén and A. Rodger, J. Am. Chem. Soc., 1990, 112, 1971-1982.

4 A. Rodger, I. S. Blagbrough, G. Adlam and M. L. Carpenter, Biopolymers, 1994, 34, 1583-1593.

5 D. Z. Coggan, I. S. Haworth, P. J. Bates, A. Robinson and A. Rodger, Inorg. Chem., 1999, 38, 4486-4497.

6 R. Marrington, T. R. Dafforn, D. J. Halsall, M. Hicks and A. Rodger, Analyst, 2005, 130, 1608-1616.

7 M. Ardhammar, N. Mikati and B. Nordén, J. Am. Chem. Soc., 1998, 120, 9957-9958.

8 A. Rodger, J. Rajendra, R. Marrington, M. Ardhammar, B. Nordén, J. D. Hirst, A. T. B. Gilbert, T. R. Dafforn, D. J. Halsall, C. A. Woolhead, C. Robinson, T. J. Pinheiro, J. Kazlauskaite, M. Seymour, N. Perez and M. J. Hannon, Phys. Chem. Chem. Phys., 2002, 4, 4051-4057.

9 M. Hicks, T. Dafforn, A. Damianoglou, P. Wormell, A. Rodger and S. Hoffman, Analyst, 2009, 134, 1623-1628.

10 E. Small, R. Marrington, A. Rodger, D. J. Scott, K. Sloan, D. Roper, T. R. Dafforn and S. G. Addinall, J. Mol. Biol., 2007, 369, 211-221.

11 R. Marrington, M. Seymour and A. Rodger, Chirality, 2006, 18, 680-690.

12 J. Rajendra and A. Rodger, Chem. - Eur. J., 2005, 11, 48414848.

13 Y. Zou, K. Razmkhah, N. P. Chmel, I. W. Hamley and A. Rodger, $R S C$ Adv. , 2013, 3, 10854-10858.
14 B. Nordén, A. Rodger and T. R. Dafforn, Linear dichroism and circular dichroism: a textbook on polarized spectroscopy, Royal Society of Chemistry, Cambridge, 2010.

15 M. A. Ismail, K. J. Sanders, G. C. Fennel, H. C. Latham, P. Wormell and A. Rodger, Biopolymers, 1998, 46, 127143.

16 E. W. Thulstrup, J. Michl and J. H. Eggers, Phys. Chem., 1970, 74, 3878-3884.

17 A. Wirtz, C. Hofmann and E. Groenen, ChemPhysChem, 2011, 12, 1519-1528.

18 E. W. Thulstrup, J. Michl and J. H. Eggers, J. Phys. Chem., 1970, 74, 3868-3877.

19 H. Juul and S. Vrønning Hoffmann, private communication, 2009.

20 D. Briggs and N. Fairley, Surf. Interface Anal., 2002, 33, 283290.

21 B. Tissington, G. Pollard and I. M. Ward, Compos. Sci. Technol., 1992, 44, 185-195.

22 S.-G. Lee, T.-J. Kang and T.-H. Yoon, J. Adhes. Sci. Technol., 1998, 12, 731-748.

23 C. W. Bunn, Trans. Faraday Soc., 1939, 35, 482-491.

24 G. Natta and P. Corradini, Nuovo Cimento, 1960, 15, 40-51.

25 A. L. Patterson, Phys. Rev., 1939, 56, 978-982.

26 M. Kubista, B. Åkerman and B. Albinsson, J. Am. Chem. Soc., 1989, 111, 7031-7035.

27 D. M. Friedrich, R. Mathies and A. C. Albrecht, J. Mol. Spectrosc., 1974, 51, 166-188.

28 J. Michl, E. W. Thulstrup and J. H. Eggers, Ber. Bunsen-Ges. Phys. Chem., 1974, 78, 575-586.

29 E. A. Chandross, J. Ferguson and E. G. McRae, J. Chem. Phys., 1966, 45, 3546-3553.

30 A. M. Mathieson, J. M. Robertson and V. C. Sinclair, Acta Crystallogr., 1950, 3, 245.

31 M. Kasha, H. R. Rawls and M. Ashraf El-Bayoumi, Pure Appl. Chem., 1965, 11, 371-392.

32 A. M. Mathieson, J. M. Robertson and V. C. Sinclair, Acta Crystallogr., 1950, 3, 245-250. 